Supporting Information for: "Understanding the Role of Overpotentials in Lithium Ion Conversion Reactions: Vis ualizing the Interface"

Guennadi Evmenenko, ${ }^{1}$ Robert E. Warburton, ${ }^{2}$ Handan Yildirim, ${ }^{2}$ Jeffrey P. Greeley, ${ }^{2}$ Maria K.Y. Chan, ${ }^{3}$ D. Bruce Buchholz, ${ }^{1}$ Paul Fenter, ${ }^{3}$ Michael J. Bedzyk, ${ }^{1}$ Timothy T. Fister ${ }^{3}$

1. Northwestern University, Evanston, IL 60208 USA

2. Purdue University, West Lafayette, IN 47907 USA

3. Argonne National Laboratory, Lemont, IL 60439 USA 


\section{Electrochemical Cell}

(a)

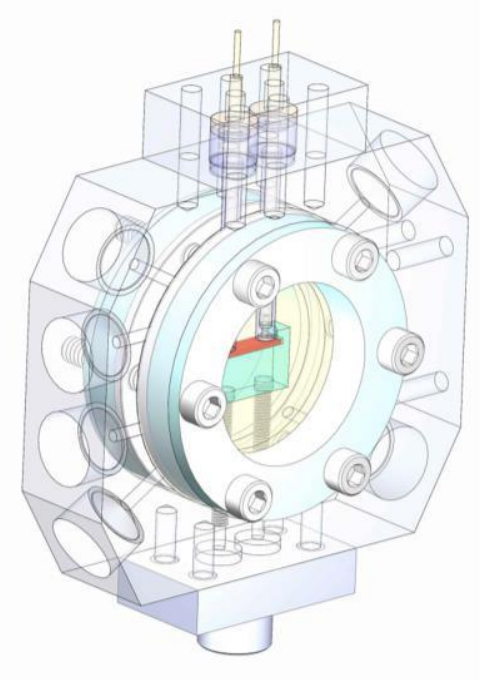

(b)

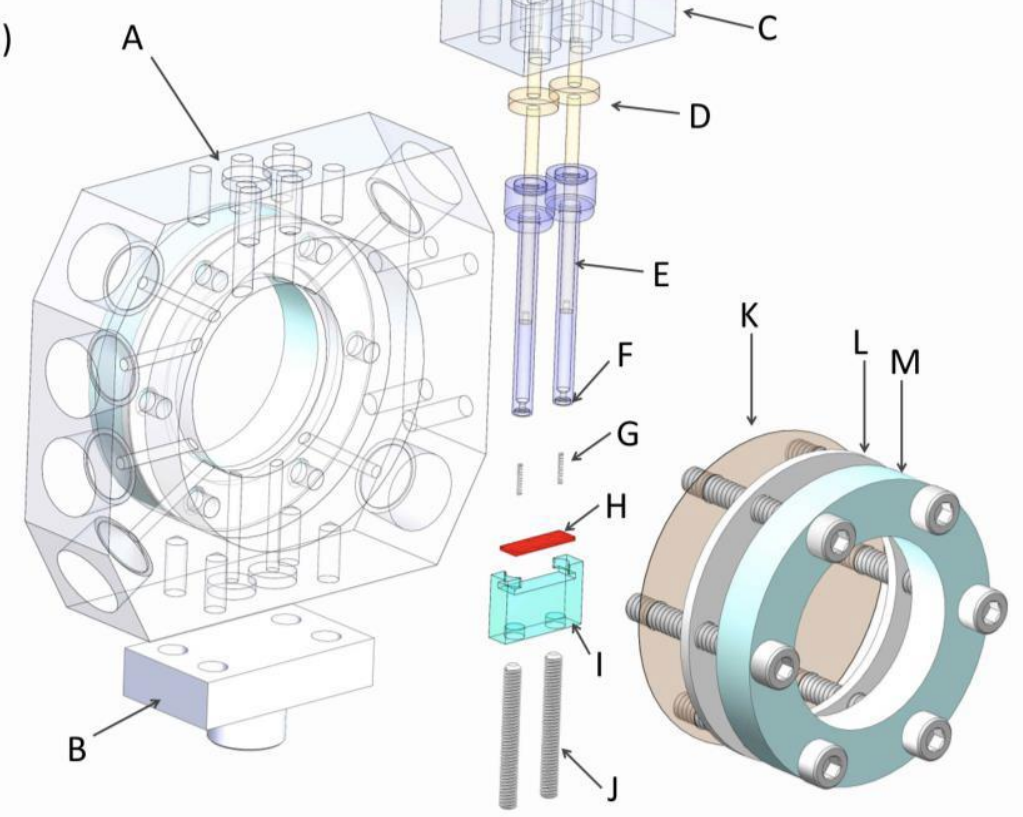

Figure S1. Electrochemical X-ray Cell. (a) Fully assembled cell and (b) exploded view of cell with each part label A-M. This cell is referred to as a 'transmission-geometry' cell because the X-rays pass through the circular opening in the cell. The sample is fully immersed in the1:1 $\mathrm{EC} / \mathrm{DMC}+1 \mathrm{M} \mathrm{LiClO}_{4}$ electrolyte, in analogy with a traditional beaker electrochemical cell. The individual labeled parts are: (A) Kel-f cell body; (B) Al goniometer adaptor; (C) Kel-f working electrode clamp; (D) stainless steel working electrode plungers; (E) Kel-f plunger shells; (F) 20-30 kfm O-ring (PSI); (G) 0.024 in. OD stainless steel compression spring (Lee, CIM010ZA); (H) 10x3x1 $\mathrm{mm}^{3}$ sample; (I) Kel-f sample holder; (J) 2-56 stainless steel set screw for sample height adjustment; (K) doubled $75 \mu \mathrm{m}$ Kapton windows; (L) Teflon flange; (M) Kel-f window clamp.

The electrolyte inlet and outlet (syringe + stopcock + Teflon tubing, not shown) are attached via PVDF compression fittings on the cell body's NPT fittings. The counter/reference electrode is a piece of lithium foil crimped to a copper wire and laid above the sample in the cell. The copper wire is epoxied to glass tubing (to seal from outside air) and attached using a compression fitting. The other NPT fittings are capped, but could be used for additional electrodes or a bubbler.

The working electrode has a primary O-ring seal at the sample using a custom miniature O-ring and secondary O-ring seals at the working clamp. The cell is mostly assembled outside of the glovebox; the lithium metal, windows, and electrolyte are attached inside an Ar-filled glovebox $\left(\mathrm{O}_{2}<1 \mathrm{ppm}\right)$. 
Analog voltages corresponding to the potential and current from the $\mathrm{CHI}$ potentiostat were saved in the SPEC file used to record the XR data.

\section{Reflectivity Fits}

Table S1. Mass densities $(\rho)$ and calculated electron densities $\left(\rho_{\mathrm{e}}\right)$ of components of the $\mathrm{Ni} / \mathrm{NiO}$ multilayer electrode.

\begin{tabular}{llll}
\hline Compound & $\begin{array}{l}\text { Density } \\
\rho\left(\mathrm{g} / \mathrm{cm}^{3}\right)\end{array}$ & $\begin{array}{l}\text { X-ray scattering Length } \\
\text { Density } \\
\left(20 \mathrm{keV}, \times 10^{-5} \mathrm{e}^{-\AA^{-2}}\right)\end{array}$ & $\begin{array}{l}\text { Effective electron } \\
\text { density }(20 \mathrm{keV}) \rho_{\mathrm{e}}\left(\mathrm{e}^{-}\right. \\
\left.\AA^{-3}\right)^{*}\end{array}$ \\
\hline $1 \mathrm{M} \mathrm{LiPF}_{6}$ & 1.2 & 1.07 & 0.38 \\
$\mathrm{EC} / \mathrm{DMC}(1: 1 \mathrm{v} / \mathrm{v})$ & 6.67 & 5.51 & 1.95 \\
$\mathrm{NiO}$ & 8.90 & 7.30 & 2.59 \\
$\mathrm{Ni}$ & 2.01 & 1.60 & 0.57 \\
$\mathrm{LiO}$ & 3.97 & 3.31 & 1.17 \\
\hline sapphire & & & \\
\hline
\end{tabular}

The scattering length density of each layer at $20 \mathrm{keV}$ was calculated using Motofit. In the forward scattering limit, the electron density can be calculated by dividing the SLD by the classical electron radius $\left(2.82 \times 10^{-5} \AA\right)$.

\section{Bilayer reflectivity fits}

Fits were performed using genetic model followed by Levenberg-Marquadt optimization of $\chi^{2}$ in Motofit. ${ }^{1}$ Statistical errorbars were found to undervalue the overall accurarcy of the measurement. Based on observed variance in the XR at high $q$, we adopted a minimum error bar of $5 \%$ for the entire measurement. Each interface in the electron density prole is calculated using an error function, e.g.

$$
\rho_{t o t}(z)=\rho_{0}+\sum_{i=1}^{N}\left(\rho_{i}-\rho_{i-1}\right)\left[1+\operatorname{erf}\left(\frac{z-z_{i}}{\sqrt{2} \sigma_{i, i-1}}\right)\right]
$$


for an $N$-layer system with top density $\rho_{0}$ (the electrolyte) and substrate density $\rho_{N}$ and interfacial roughness $\sigma_{i, i-1}$. An example of how multiple layers form an overall density profile is given in fits below. The parameters used in the each fit are shown in the accompanying tables.

As discussed by Nelson[5], the reflectivity, $R$, is calculated from these parameters using a matrix approach functionally equivalent to Parrat's recursive method for a stratified thin film.

$$
R=\left|\frac{M_{11}}{M_{21}}\right|^{2}, \text { where } M=\prod_{i=0}^{N} c_{i, i+1}
$$

Here, the the ith layer is represented as a characteristic matrix $c i$ made up of terms consisting of Fresnel coefficients $r i ; i+1$ and phase factors $\beta_{i, i+1}=k_{i}\left(z_{i+1}-z_{i}\right)$,

$$
c_{i, i+1}=\left(\begin{array}{cc}
e^{\beta i, i+1} & r_{i, i+1} e^{\beta_{i, i+1}} \\
r_{i, i+1} e^{-\beta_{i, i+1}} & e^{\beta i, i+1}
\end{array}\right)
$$

where $r_{i, i+1}=\frac{k_{i}-k_{i+1}}{k_{i}+k_{i+1}}$ and $k_{i}=\sqrt{4 \pi^{2}-4 \pi\left(\rho_{i}-\rho_{0}\right)}$.

Fits were evaluated by find a minimum reduced $\chi^{2}$ for a minimally covarying $\rho(z)$. Error bars of $\rho(z)$ were calculated using the covariance matrix $V_{i, j}$ and standard deviations, $\sigma_{i}$, for each parameter, $x_{i}$ :

$$
\sigma_{\rho}(z)=\left[\sum_{i, j} V_{i, j} \sigma_{i} \sigma_{j} \frac{\partial \rho}{\partial x_{i}} \frac{\partial \rho}{\partial x_{j}}\right]^{1 / 2}
$$

\section{Bilaye r fits}

Fits used independent variables defining each slab (thickness, density, interfacial roughensses). Scaling factor for the data was fixed. Background was set to zero (since background was subtracted from original data). Imaginary part of the density was also set to zero since absorption was minimal for these films. The sapphire substrate and electrolyte (superstrate) layers were fixed to values found in Table S1. 


\begin{tabular}{|l|l|l|}
\hline \multicolumn{3}{|l|}{ Bilayer 3.1 V (fit1), $\chi^{2}=61$} \\
\hline Thickness $(\AA)$ & Density $\left(1 / \AA^{3}\right)$ & Roughness $(\AA)$ \\
\hline Electrolyte & 0.38 & 0.00 \\
\hline $14.36 \pm 0.01$ & $1.966 \pm 0.004$ & $2.498 \pm 0.006$ \\
\hline $27.23 \pm 0.01$ & $2.597 \pm 0.004$ & $1.851 \pm 0.010$ \\
\hline Substrate & 1.19 & $1.407 \pm 0.003$ \\
\hline
\end{tabular}
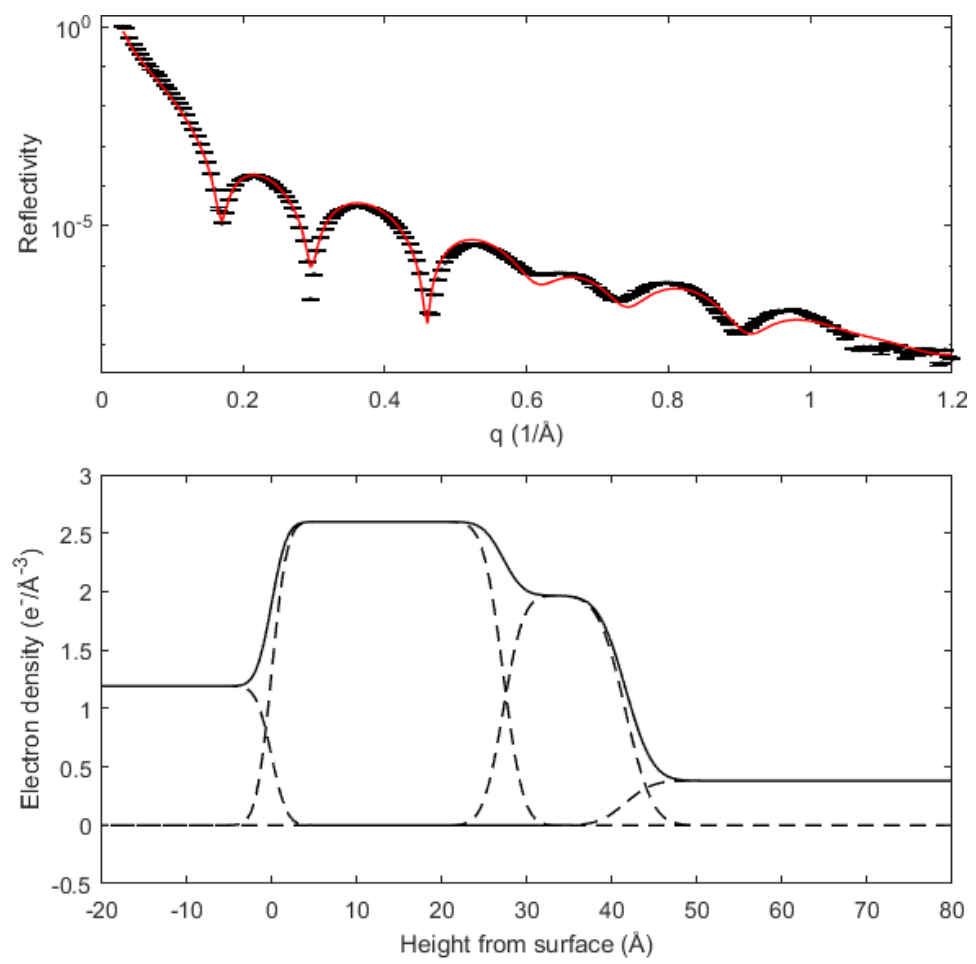

\begin{tabular}{|l|l|l|}
\hline \multicolumn{3}{|l|}{ Bilayer $2.2 \mathrm{~V}, \chi^{2}=21.2$} \\
\hline Thickness $(\AA)$ & Density $\left(1 / \AA^{3}\right)$ & Roughness $(\AA)$ \\
\hline Electrolyte & 0.38 & 0.00 \\
\hline $9.62 \pm 0.01$ & $1.712 \pm 0.006$ & $2.309 \pm 0.010$ \\
\hline $1.08 \pm 0.03$ & $0.122 \pm 0.047$ & $2.120 \pm 0.018$ \\
\hline $31.16 \pm 0.04$ & $2.517 \pm 0.003$ & $3.864 \pm 0.022$ \\
\hline Substrate & 1.19 & $1.315 \pm 0.003$ \\
\hline
\end{tabular}
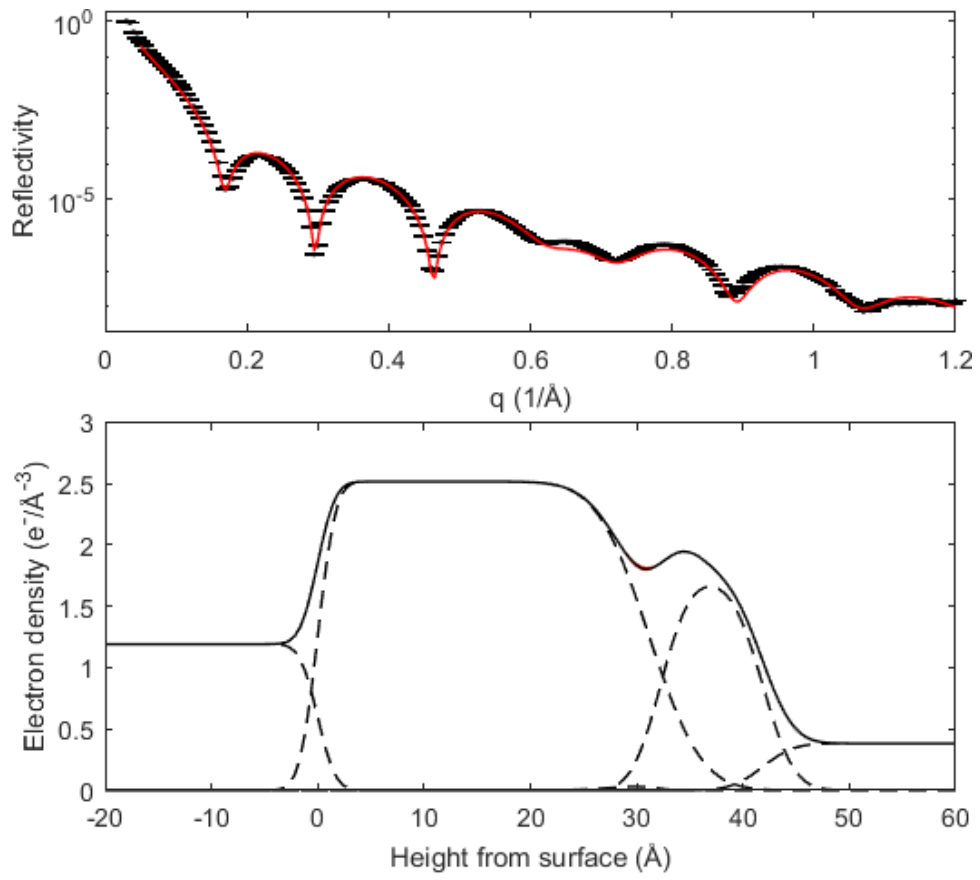


\begin{tabular}{|l|l|l|}
\hline \multicolumn{3}{|l|}{ Bilayer $1.9 \mathrm{~V}$ (fit1), $\chi^{2}=9.3$} \\
\hline Thickness $(\AA)$ & Density $\left(1 / \AA^{3}\right)$ & Roughness $(\AA)$ \\
\hline Electrolyte & 0.38 & 0.00 \\
\hline $9.47 \pm 0.03$ & $1.485 \pm 0.013$ & $3.043 \pm 0.029$ \\
\hline $1.05 \pm 0.02$ & $-1.769 \pm 0.050$ & $1.831 \pm 0.009$ \\
\hline $32.71 \pm 0.02$ & $2.597 \pm 0.004$ & $3.788 \pm 0.018$ \\
\hline Substrate & 1.19 & $1.234 \pm 0.004$ \\
\hline
\end{tabular}
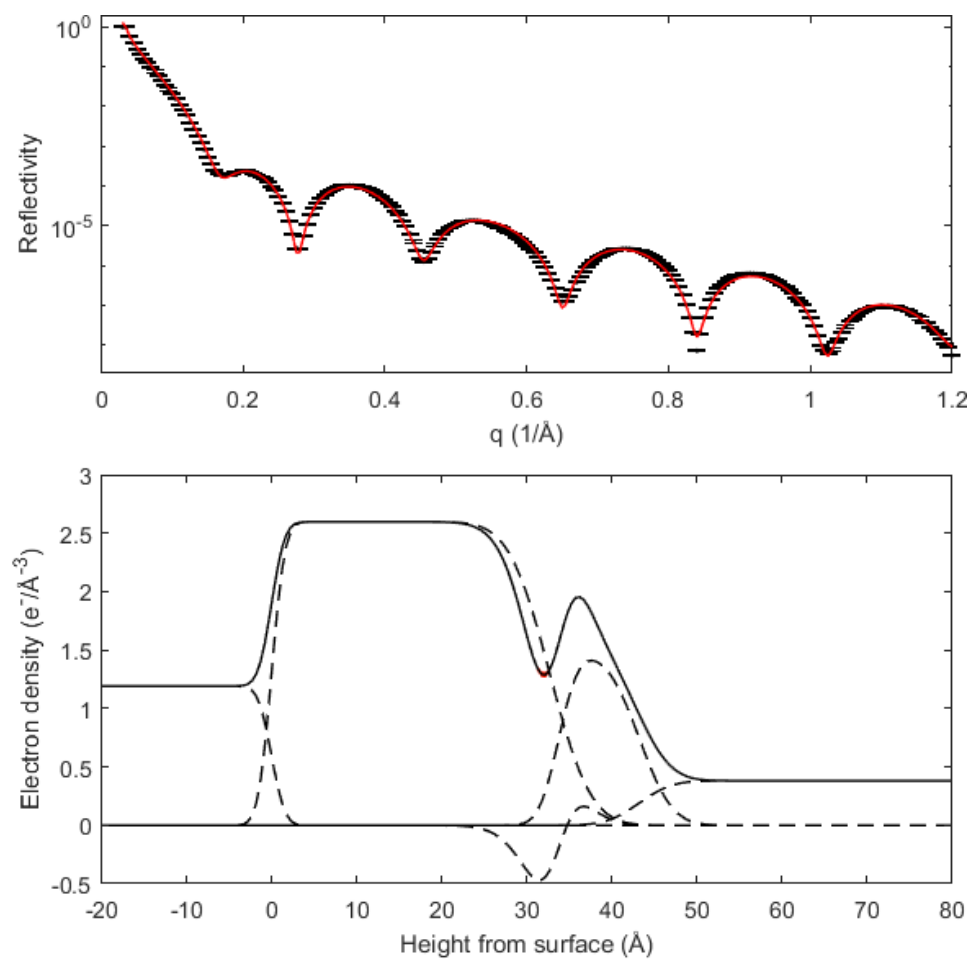

\begin{tabular}{|l|l|l|}
\hline \multicolumn{2}{|l|}{ Bilayer $1.2 \mathrm{~V}(\mathrm{fit} 4), \chi^{2}=48$} \\
\hline Thickness $(\AA)$ & Density $\left(1 / \AA^{3}\right)$ & Roughness $(\AA)$ \\
\hline Electrolyte & 0.38 & 0.00 \\
\hline $8.76 \pm 0.08$ & $1.411 \pm 0.022$ & $3.428 \pm 0.058$ \\
\hline $1.78 \pm 0.04$ & $-1.438 \pm 0.053$ & $1.640 \pm 0.008$ \\
\hline $33.13 \pm 0.04$ & $2.633 \pm 0.004$ & $3.810 \pm 0.023$ \\
\hline Substrate & 1.19 & $1.460 \pm 0.005$ \\
\hline
\end{tabular}
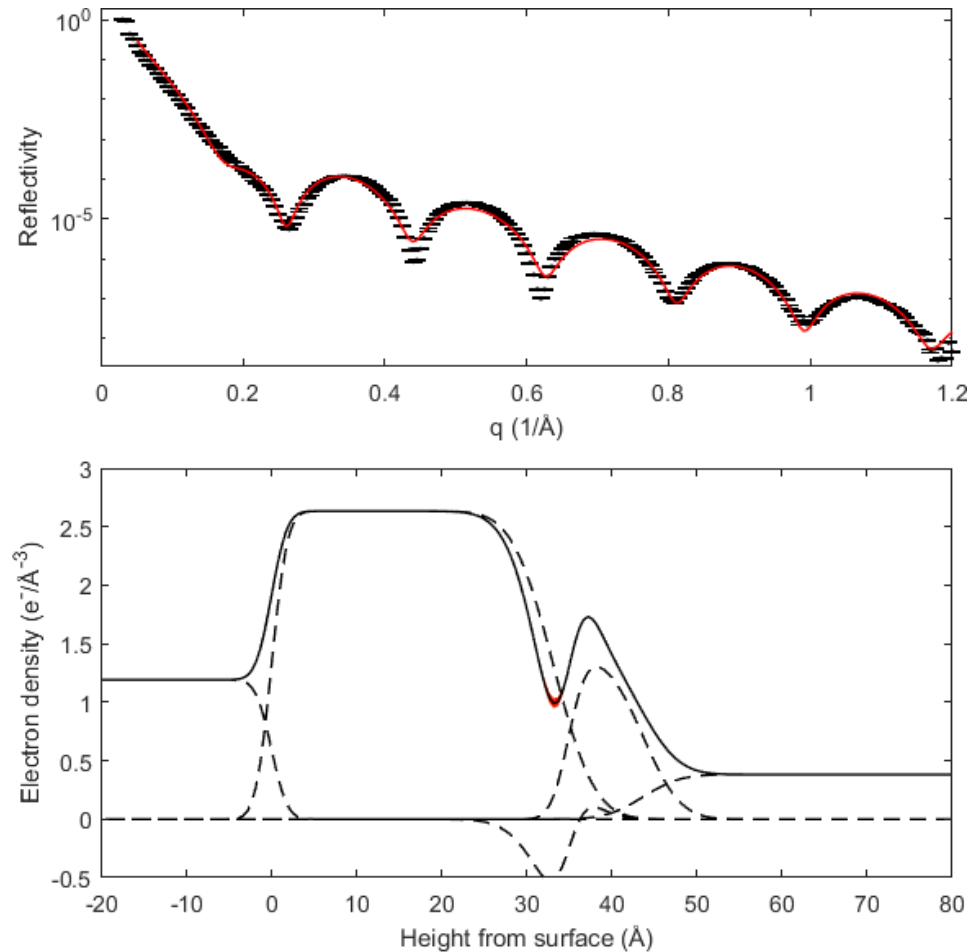


\begin{tabular}{|l|l|l|}
\hline \multicolumn{3}{|l|}{ Bilayer 0.7 V (fit1), $\chi^{2}=37.5$} \\
\hline Thickness $(\AA)$ & Density $\left(1 / \AA^{3}\right)$ & Roughness $(\AA)$ \\
\hline Electrolyte & 0.38 & 0.00 \\
\hline $14.00 \pm 0.04$ & $1.607 \pm 0.008$ & $3.786 \pm 0.056$ \\
\hline $4.78 \pm 0.05$ & $0.981 \pm 0.015$ & $1.259 \pm 0.033$ \\
\hline $31.11 \pm 0.01$ & $2.610 \pm 0.005$ & $1.259 \pm 0.019$ \\
\hline Substrate & 1.19 & $1.841 \pm 0.006$ \\
\hline
\end{tabular}
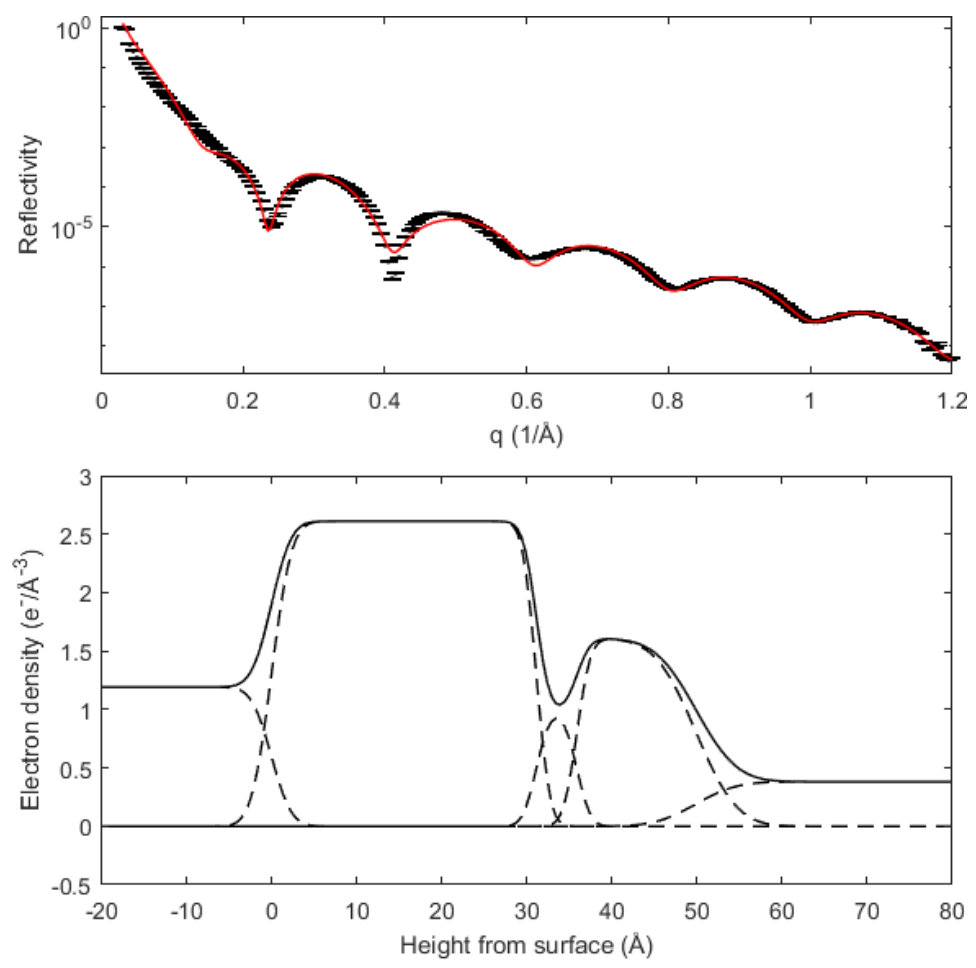

\begin{tabular}{|l|l|l|}
\hline \multicolumn{2}{|l|}{ Bilayer $0.3 \mathrm{~V}$ (fit4), $\chi^{2}=14.0$} \\
\hline Thickness $(\AA)$ & Density $\left(1 / \AA^{3}\right)$ & Roughness $(\AA)$ \\
\hline Electrolyte & 0.38 & 0.00 \\
\hline $21.97 \pm 0.25$ & $1.241 \pm 0.013$ & $7.861 \pm 0.095$ \\
\hline $2.06 \pm 0.05$ & $-1.160 \pm 0.087$ & $1.484 \pm 0.016$ \\
\hline $32.23 \pm 0.05$ & $2.706 \pm 0.006$ & $2.495 \pm 0.023$ \\
\hline Substrate & 1.19 & $1.848 \pm 0.007$ \\
\hline
\end{tabular}
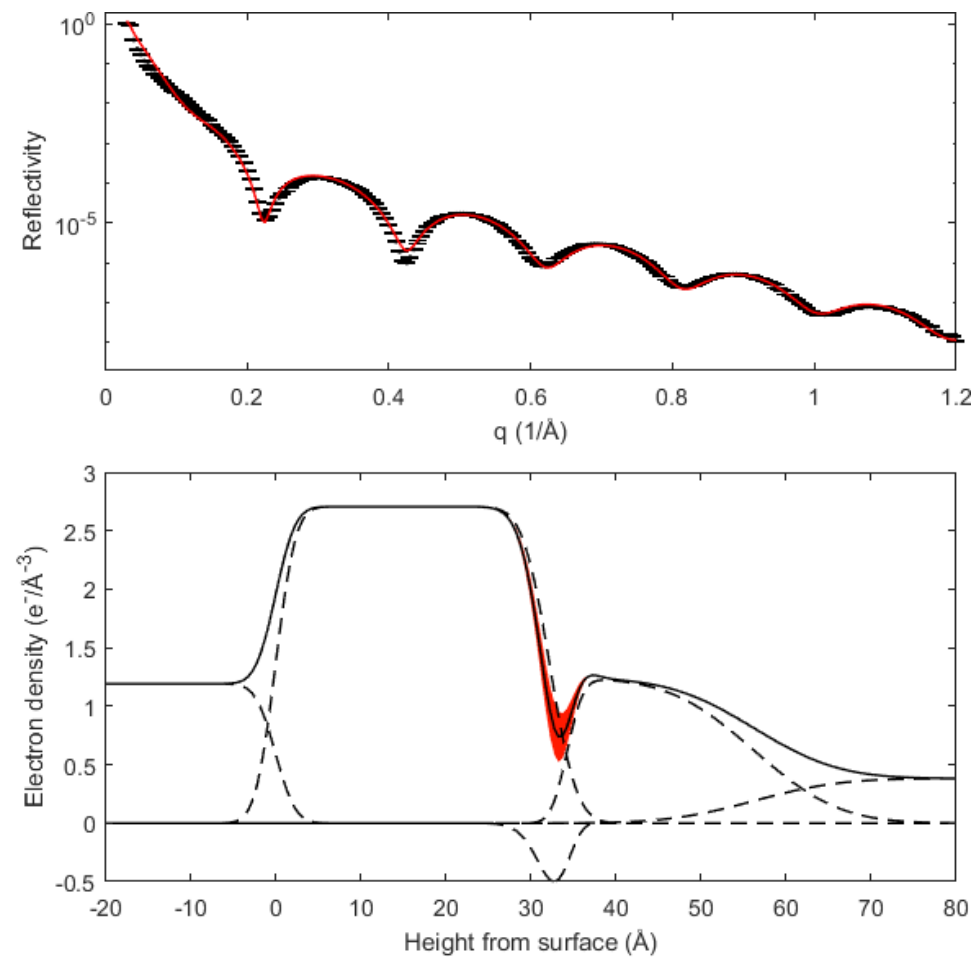

\section{Trilayer fits}


Three layers was found to not reproduce the large Kiessig fringes at high-Q. A four layer model, with a thin top layer gave a starkly better $\chi^{2}$ without significant covariance. We believe the top layer represents the native oxide of the outer nickel layer.

\begin{tabular}{|l|l|l|}
\hline \multicolumn{2}{|l|}{ Trilayer 3.1 V (fit9), $\chi^{2}=25$} \\
\hline Thickness $(\AA)$ & Density $\left(1 / \AA^{3}\right)$ & Roughness $(\AA)$ \\
\hline Electrolyte & 0.38 & 0.00 \\
\hline $0.03 \pm 0.01$ & $-0.696 \pm 0.015$ & $1.640 \pm 0.000$ \\
\hline $16.65 \pm 0.03$ & $2.698 \pm 0.006$ & $3.165 \pm 0.012$ \\
\hline $13.48 \pm 0.03$ & $1.795 \pm 0.005$ & $2.713 \pm 0.022$ \\
\hline $28.85 \pm 0.02$ & $2.562 \pm 0.004$ & $2.227 \pm 0.015$ \\
\hline Substrate & 1.19 & $1.245 \pm 0.004$ \\
\hline
\end{tabular}
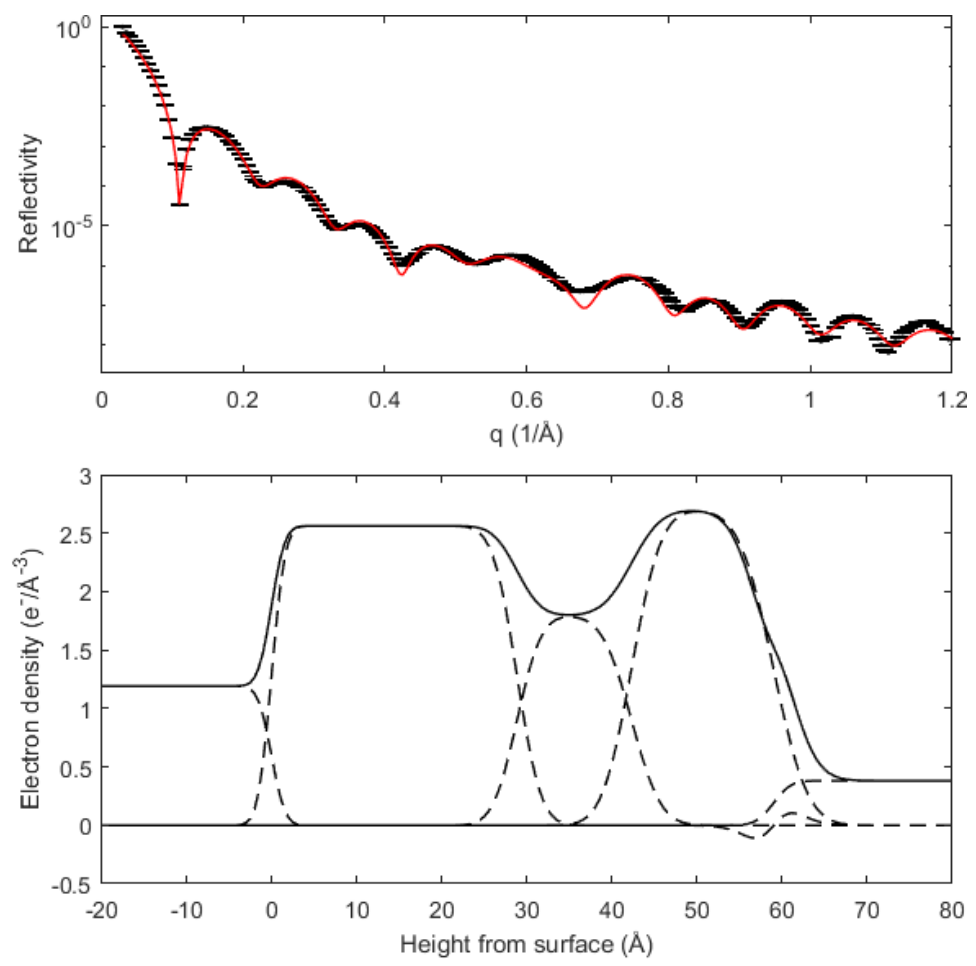

\begin{tabular}{|l|l|l|}
\hline \multicolumn{2}{|l|}{ Trilayer $2.2 \mathrm{~V}$ (fit4), $\chi^{2}=12.1$} \\
\hline Thickness $(\AA)$ & Density $\left(1 / \AA^{3}\right)$ & Roughness $(\AA)$ \\
\hline Electrolyte & 0.38 & 0.00 \\
\hline $3.11 \pm 0.03$ & $-0.948 \pm 0.022$ & $1.585 \pm 0.013$ \\
\hline $17.76 \pm 0.03$ & $2.574 \pm 0.008$ & $3.908 \pm 0.011$ \\
\hline $9.46 \pm 0.02$ & $1.761 \pm 0.008$ & $1.977 \pm 0.013$ \\
\hline $2.70 \pm 0.00$ & $1.035 \pm 0.012$ & $1.569 \pm 0.014$ \\
\hline $30.28 \pm 0.01$ & $2.588 \pm 0.004$ & $2.831 \pm 0.019$ \\
\hline Substrate & 1.19 & $1.271 \pm 0.004$ \\
\hline
\end{tabular}
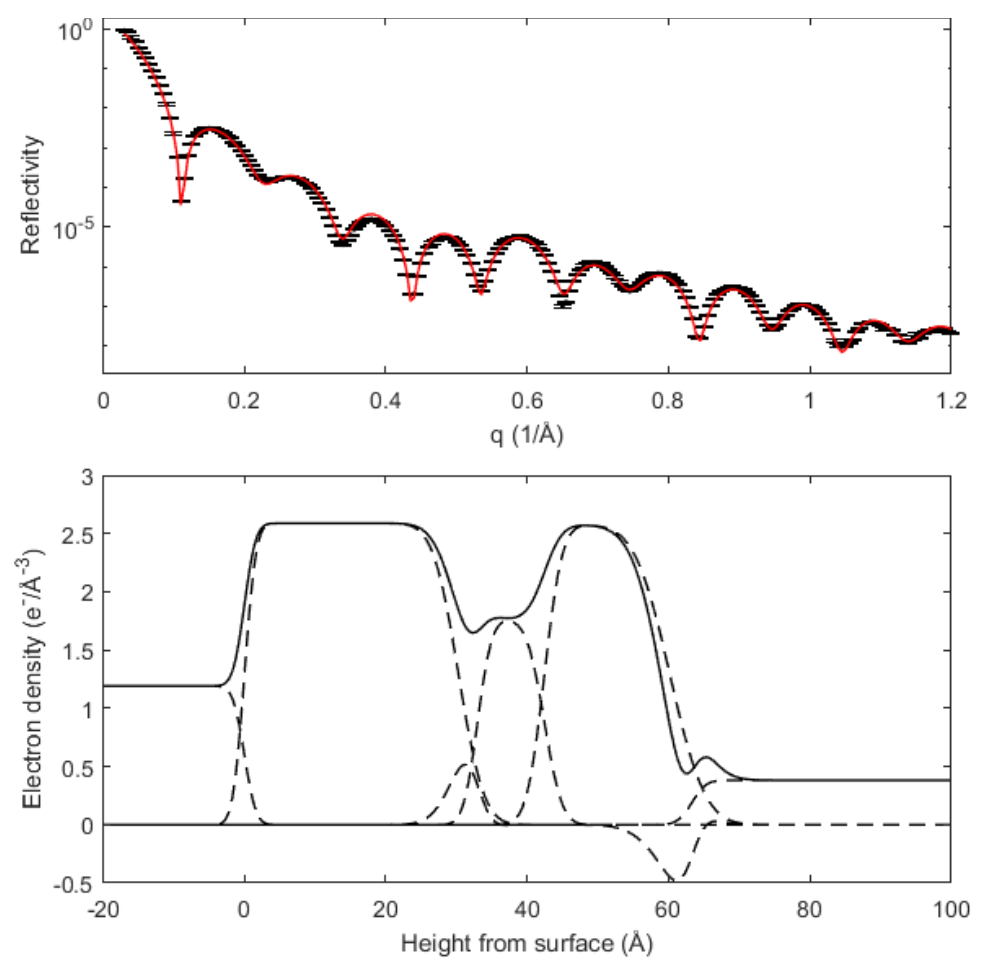


\begin{tabular}{|l|l|l|}
\hline \multicolumn{2}{|l|}{ Trilayer $1.9 \mathrm{~V}$ (fit4), $\chi^{2}=28$} \\
\hline Thickness $(\AA)$ & Density $\left(1 / \AA^{3}\right)$ & Roughness $(\AA)$ \\
\hline Electrolyte & 0.38 & 0.00 \\
\hline $1.80 \pm 0.05$ & $-2.093 \pm 0.067$ & $2.034 \pm 0.019$ \\
\hline $20.15 \pm 0.04$ & $2.648 \pm 0.008$ & $3.747 \pm 0.016$ \\
\hline $6.99 \pm 0.11$ & $1.055 \pm 0.009$ & $1.808 \pm 0.009$ \\
\hline $4.97 \pm 0.09$ & $1.692 \pm 0.012$ & $3.000 \pm 0.000$ \\
\hline $4.90 \pm 0.07$ & $1.171 \pm 0.028$ & $0.663 \pm 0.064$ \\
\hline $29.87 \pm 0.04$ & $2.619 \pm 0.004$ & $1.901 \pm 0.039$ \\
\hline Substrate & 1.19 & $1.197 \pm 0.005$ \\
\hline
\end{tabular}
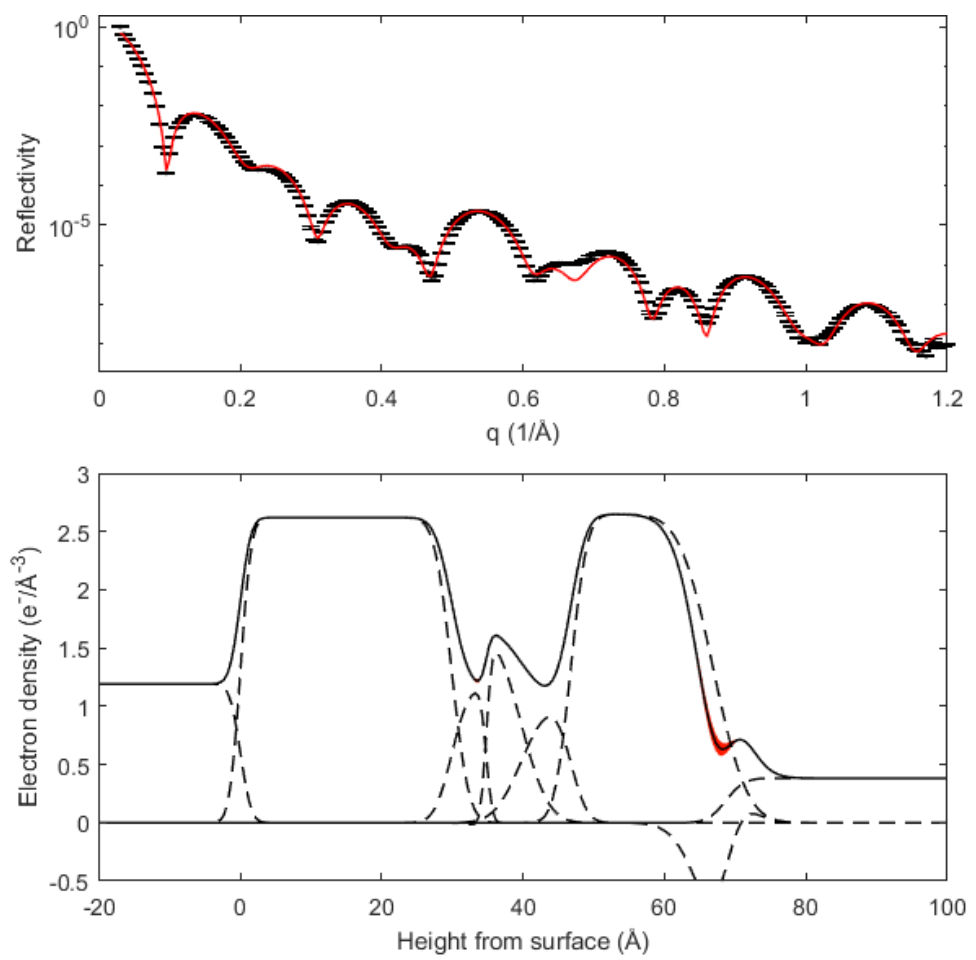

\begin{tabular}{|l|l|l|}
\hline \multicolumn{3}{|l|}{ Trilayer 0.8 V (fit2), $\chi^{2}=41$} \\
\hline Thickness $(\AA)$ & Density $\left(1 / \AA^{3}\right)$ & Roughness $(\AA)$ \\
\hline Electrolyte & 0.38 & 0.00 \\
\hline $2.43 \pm 0.05$ & $-1.033 \pm 0.038$ & $2.293 \pm 0.021$ \\
\hline $21.88 \pm 0.05$ & $2.577 \pm 0.009$ & $5.315 \pm 0.033$ \\
\hline $11.38 \pm 0.02$ & $0.988 \pm 0.007$ & $2.368 \pm 0.010$ \\
\hline $6.40 \pm 0.01$ & $1.403 \pm 0.009$ & $1.329 \pm 0.027$ \\
\hline $2.79 \pm 0.00$ & $-0.339 \pm 0.018$ & $1.818 \pm 0.010$ \\
\hline $32.71 \pm 0.01$ & $2.612 \pm 0.005$ & $2.935 \pm 0.020$ \\
\hline Substrate & 1.19 & $1.214 \pm 0.004$ \\
\hline
\end{tabular}
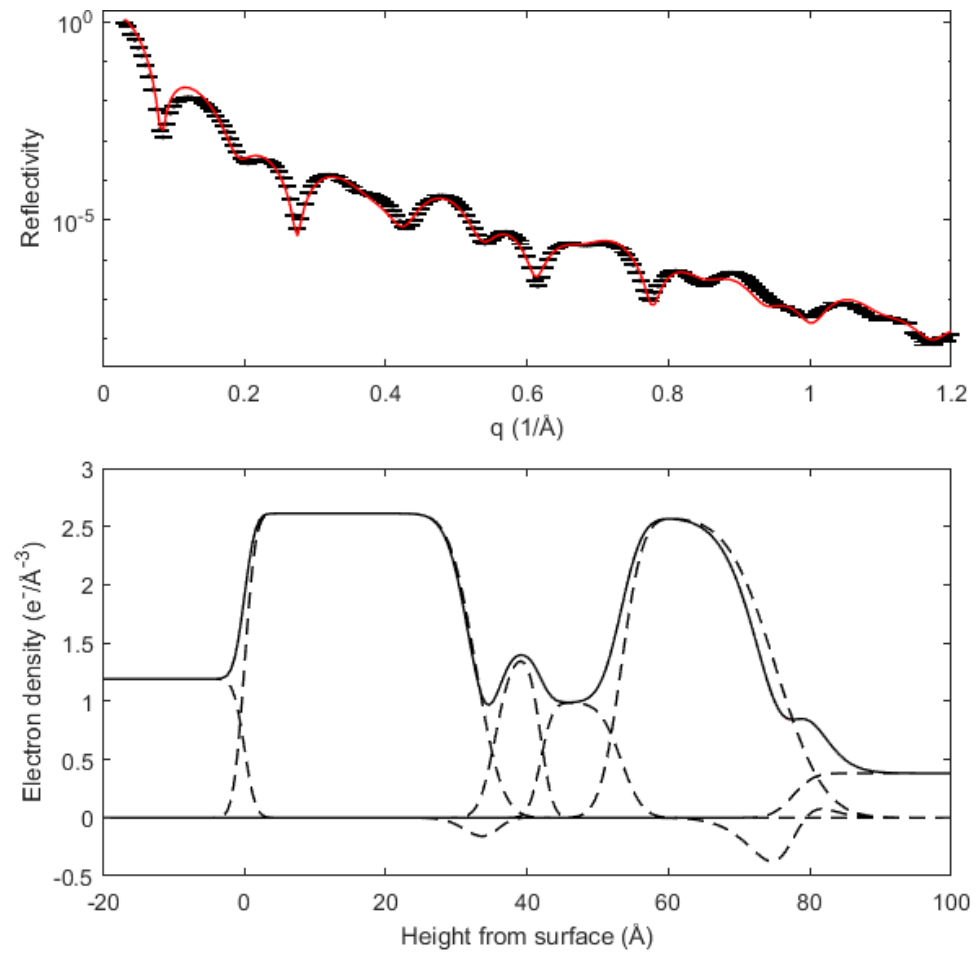


\begin{tabular}{|l|l|l|}
\hline \multicolumn{2}{|l|}{ Trilayer 0.7 V (fit1), $\chi^{2}=48$} \\
\hline Thickness $(\AA)$ & Density $\left(1 / \AA^{3}\right)$ & Roughness $(\AA)$ \\
\hline Electrolyte & 0.38 & 0.00 \\
\hline $3.73 \pm 0.08$ & $-0.690 \pm 0.032$ & $2.127 \pm 0.024$ \\
\hline $20.14 \pm 0.08$ & $2.638 \pm 0.011$ & $5.465 \pm 0.048$ \\
\hline $11.68 \pm 0.03$ & $0.822 \pm 0.009$ & $2.462 \pm 0.010$ \\
\hline $11.91 \pm 0.03$ & $1.368 \pm 0.010$ & $2.029 \pm 0.026$ \\
\hline $2.70 \pm 0.00$ & $-0.136 \pm 0.017$ & $3.273 \pm 0.042$ \\
\hline $31.66 \pm 0.01$ & $2.580 \pm 0.006$ & $2.056 \pm 0.006$ \\
\hline Substrate & 1.19 & $1.135 \pm 0.004$ \\
\hline
\end{tabular}
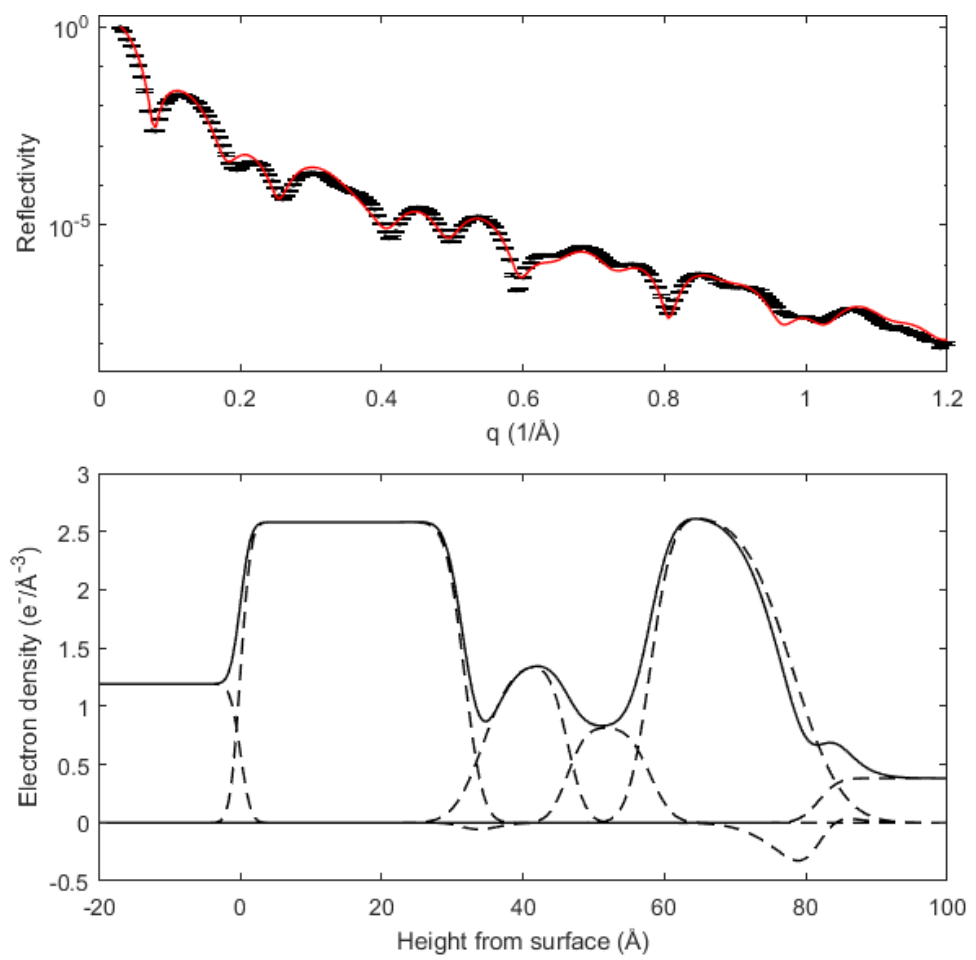

\section{Class ical molecular dynamics simulations}

Classical molecular dynamics simulations are performed using the DL_POLY code. ${ }^{2}$ A core-shell potential is fit using parameters from Hartree-Fock calculations. The MD simulations are performed on a supercell containing $500 \mathrm{Li}_{2} \mathrm{O}$ formula units (1500 total atoms) and we confirm a melt of $\mathrm{Li}_{2} \mathrm{O}$ to an amorphous phase by the radial distribution functions (RDF) in Figure S2a. The RDF, along with the structural model (Figure S3b) demonstrate the formation of an amorphous phase with a density of $1.99 \mathrm{~g}$ $\mathrm{cm}^{-3}$. Density functional geometry relaxations of 72 and 144 atom models cut from the amorphous cell suggest an average $\mathrm{Li}_{2} \mathrm{O}$ amorphization energy of $0.44 \mathrm{eV}$ per $\mathrm{Li}_{2} \mathrm{O}$ formula unit. This amorphous reference state is applied for the 'bulk' $\mathrm{Li}_{2} \mathrm{O}$ thermodynamics as described in the main text for calculation of interfacial energies and the potential-dependent bulk reaction thermodynamics. 

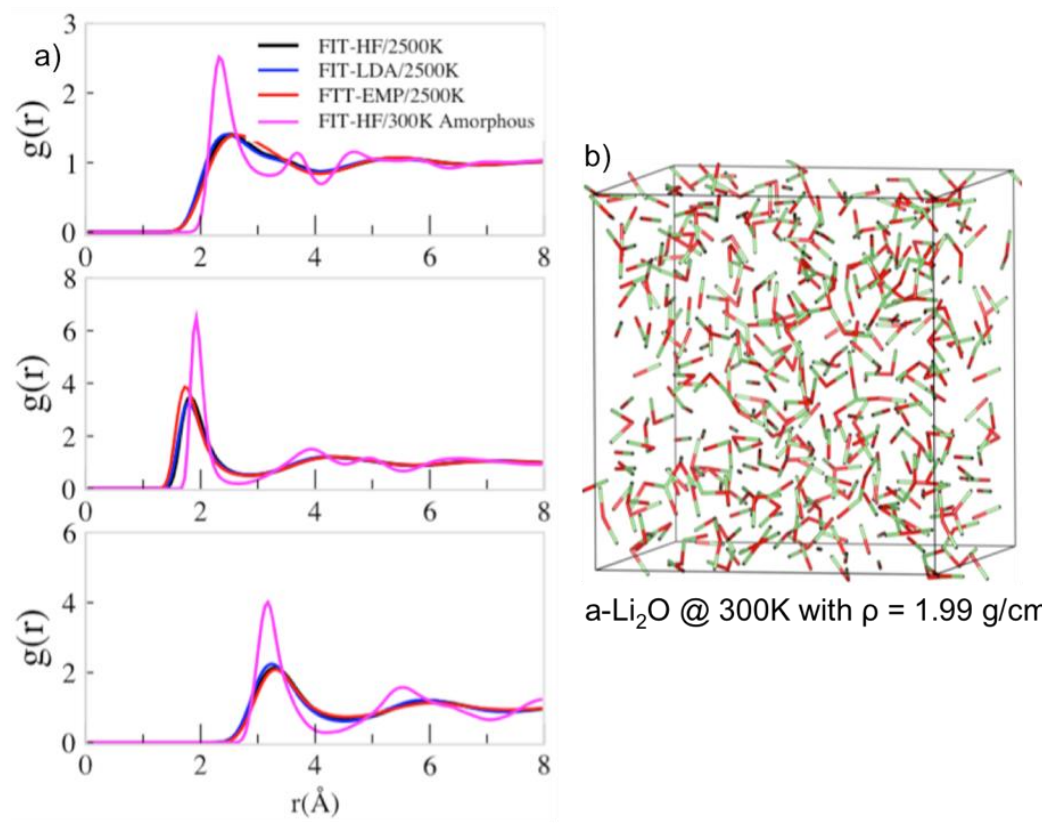

a- $\mathrm{Li}_{2} \mathrm{O} @ 300 \mathrm{~K}$ with $\rho=1.99 \mathrm{~g} / \mathrm{cm}^{3}$

Figure S2. a) Radial distribution functions for (top to bottom) $\mathrm{Li}-\mathrm{Li}, \mathrm{Li}-\mathrm{O}$, and $\mathrm{O}-\mathrm{O}$ ion pairs for the $a$ $\mathrm{Li}_{2} \mathrm{O}$ at $300 \mathrm{~K}$ (purple) and those corresponding to the melt (shown by black, blue, and red lines). b) Snapshot of $a-\mathrm{Li}_{2} \mathrm{O}$ structure obtained at $300 \mathrm{~K}$.

\section{Inte rfacial structures}

Interfacial models for $\mathrm{Ni}(111) / a-\mathrm{Li}_{2} \mathrm{O}, \mathrm{NiO}(100) / a-\mathrm{Li}_{2} \mathrm{O}$, and $\mathrm{Ni}(111) / \mathrm{NiO}(100)$ are constructed using the approach outlined in the Methods section of the main text. Representative models of the structures sampled to estimate their respective interfacial energies are shown in Figure S3. Further description of these interfaces and the nucleation model will be highlighted in a future publication. 
a)

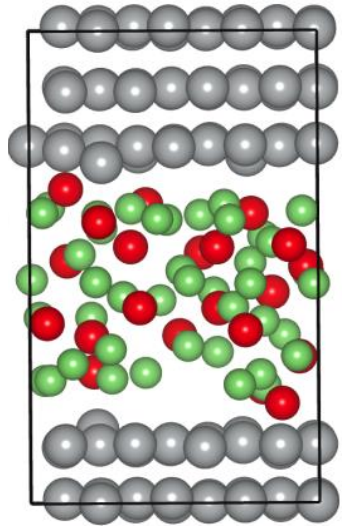

b)

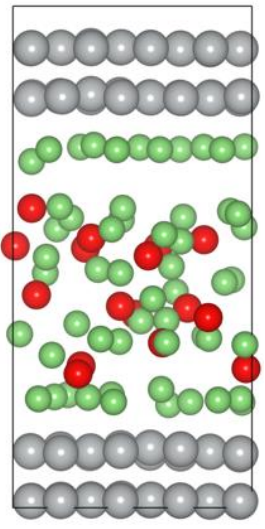

c)

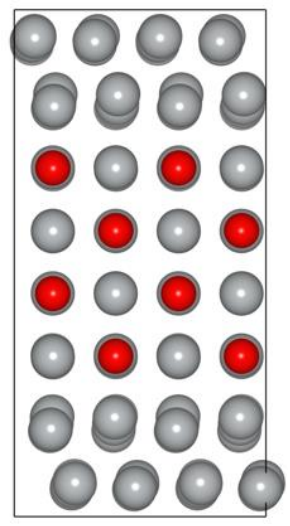

d)

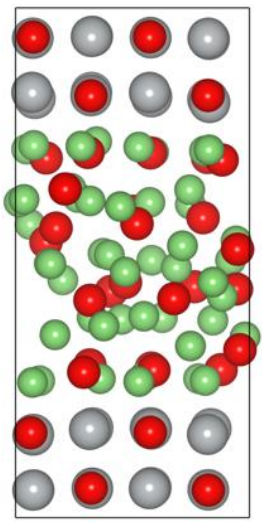

Figure S3 Interfacial models for (a) $\mathrm{Ni} / a-\mathrm{Li}_{2} \mathrm{O}$ with an interfacial energy of $1.86 \mathrm{~J} \mathrm{~m}^{-2}$, (b) $\mathrm{Ni} / \mathrm{Li} / a-\mathrm{Li}_{2} \mathrm{O}$ with an interfacial energy of $0.41 \mathrm{~J} \mathrm{~m}^{-2}$, (c) $\mathrm{Ni} / \mathrm{NiO}$ with an interfacial energy of $1.44 \mathrm{~J} \mathrm{~m}^{-2}$, and (d) $\mathrm{NiO} / a-\mathrm{Li}_{2} \mathrm{O}$ with an interfacial energy of $0.35 \mathrm{~J} \mathrm{~m}^{-2}$. In (a)-(d), $\mathrm{Ni}, \mathrm{Li}$, and $\mathrm{O}$ atoms are depicted in silver, green, and red, respectively.

\section{References}

1. Nelson, A., Co-Refinement of Multiple-Contrast Neutron/X-Ray Reflectivity Data Using Motofit. J. Appl. Crystallogr. 2006, 39, 273-276.

2. Smith, W.; Forester, T. R., DI_Poly_2.0: A General-Purpose Parallel Molecular Dynamics Simulation Package. J. Mol. Graphics 1996, 14, 136-141. 